\title{
SIGNIFICADO ESTRATIGRÁFICO DE PALINOMORFOS RETRABAJADOS EN LA FORMACIÓN MALIMÁN (VISEANO) EN LA SIERRA DEL VOLCÁN, PROVINCIA DE SAN JUAN, ARGENTINA: RESULTADOS PRELIMINARES
}

\author{
CECILIA AMENÁBAR \\ CONICET, Universidad de Buenos Aires, Departamento de Geología, Ciudad Universitaria, Pabellón 2, \\ (1428) Buenos Aires, Argentina.amenabar@gl.fcen.uba.ar
}

\begin{abstract}
RESUMO - A Formação Malimán é parte de um conjunto de sedimentos de idade viseana que afloram na serra de Volcán (bacia de Río Blanco), província de San Juan, Argentina (cerca de $30^{\circ} \mathrm{S}$ e $69^{\circ} \mathrm{O}$ ), os quais se depositam em discordância angular sobre o Grupo Chinguillos, de idade devoniana (Givetiano-Frasniano). Esta unidade apresenta uma associação palinológica composta por um conjunto de formas autóctones y outro de elementos retrabalhados (12,5-55\%), incluindo esporos e paleomicroplâncton. O objetivo deste trabalho é discutir o significado estratigráfico dos palinomorfos compreendidos no conjunto retrabalhado. Com base nas distribuições estratigráficas, reconhecem-se dois intervalos temporais: Eodevoniano (Emsiano) e Devoniano médio-tardio (Givetiano-Fameniano precoce). As formas retrabalhadas são comparadas às microfloras siluro-devonianas da Argentina e de outras bacias da América do Sul. Baseado em reconstruções paleogeográficas do Eocarbonífero da América do Sul, considera-se a Pré-Cordilheira como a principal área fonte, a qual se encontrava próxima ao depocentro da bacia. Por fim, comprova-se a existência de um hiato erosivo e/ou não deposicional na seção estudada, que compreende o intervalo Neodevoniano (Fameniano tardío) - Eocarbonífero (Tournaisiano).
\end{abstract}

Palavras-chave: Palinomorfos retrabalhados, proveniência, Eocarbonífero, Argentina.

\begin{abstract}
STRATIGRAPHIC SIGNIFICANCE OF REWORKED PALYNOMORPHS IN THE MALIMÁN FORMATION (VISEAN), FROM THE SIERRA DEL VOLCÁN, SAN JUAN PROVINCE, ARGENTINA: PRELIMINARY RESULTS. The Malimán Formation, Viséan in age, crops out at the Volcán range (Río Blanco Basin), northwestern Precordillera in San Juan province, Argentina, ca. $30^{\circ} \mathrm{S}$ and $69^{\circ} \mathrm{W}$. An erosional unconformity separates the Malimán Formation from the underlying Chigua Formation of Middle Devonian (Givetian-Frasnian). This unit bears a palynological assemblage composed of two groups of palynomorphs, one with autochthonous species and other of recycled ones (12.5-55\%), which includes spores and palaeomicroplankton. In the present contribution, the stratigraphic significance of the reworked palynomorphs is discussed. Based on their stratigraphic ranges, two source intervals are recognized: Early Devonian (Emsian) and Middle-Late Devonian (Givetian-Early Famennian). The recycled palynomorphs are similar to those of Siluro-Devonian Argentinian and South American assemblages. Based on Early Carboniferous palaeogeographic reconstructions of South America, the Precordillera is considered as the source area, which was placed near the depocentre. An erosional and/or a depositional hiatus, comprising the Late Devonian (Late Famennian) to the Early Carboniferous (Tournaisian), is confirmed for the studied section.
\end{abstract}

Key words: Reworked palynomorphs, provenance, Early Carboniferous, Argentina.

\section{INTRODUCCIÓN}

Determinar la presencia de palinomorfos retrabajados en asociaciones palinológicas de cualquier edad constituye siempre un desafío que se ve particularmente agravado cuando la diferencia temporal entre los conjuntos autóctono y retrabajado es corta (e.g., Devónico y Carbonífero). Muchas veces la preservación no ayuda a discriminar entre ambos conjuntos de palinomorfos y solamente el registro previo de las especies puede ser utilizado para separarlos.
Este hecho a su vez, torna complejo el proceso de clasificar taxonómicamente los palinomorfos de una asociación más aún si el porcentaje de retrabajo es alto (di Pasquo \& Azcuy, 1997). De allí que con frecuencia se ignore la presencia de estas formas recicladas, a pesar de que la erosión y la redepositación de partículas inorgánicas y orgánicas en sedimentos más jóvenes es un hecho muy común (e.g., Richardson \& Rasul, 1978). Sin embargo, en aquellos sedimentos donde el retrabajo es muy abundante y difícil de diferenciar del material autóctono a causa de su corta 
separación temporal, cobra mayor importancia realizar esta tarea con sumo detalle para evitar una interpretación errónea de la edad de los estratos involucrados.

En este trabajo se da a conocer la presencia de palinomorfos retrabajados presentes en 8 niveles pelíticos de la Formación Malimán (Figuras 1 y 2), en la quebrada Cortaderas (localidad tipo), Precordillera de San Juan, Argentina, de edad Carbonífero Temprano y se proponen distintas fuentes de proveniencia. Además, se discute palinológicamente la presencia de un hiato en el límite Devónico/Carbonífero. Las muestras se hallan depositadas en el Laboratorio de Palinología del Departamento de Ciencias Geológicas de la Universidad de Buenos Aires.

\section{MARCO GEOLÓGICO}

La Formación Malimán (FM), unidad inferior del Grupo Angualasto (Limarino \& Césari, 1993), es parte de un conjunto de sedimentitas de edad viseana (Rodríguez Amenábar et al., 2003) que aflora en la sierra del Volcán (Cuenca Río Blanco), provincia de San Juan, Argentina, entre los paralelos $30^{\circ}$ y $69^{\circ}$ de latitud sur. Esta unidad, con un espesor de $1300 \mathrm{~m}$, se apoya en discordancia angular sobre la Formación Chigua (Devónico Medio), e infrayace en discordancia erosiva a la Formación Cortaderas (Figura 1). En ella se reconocieron la Palinozona Verrucosisporites-Cordylosporites (Césari \& Gutierrez, 2000), las Fitozonas Archaeosigillaria-Malimanium y Frenguellia-

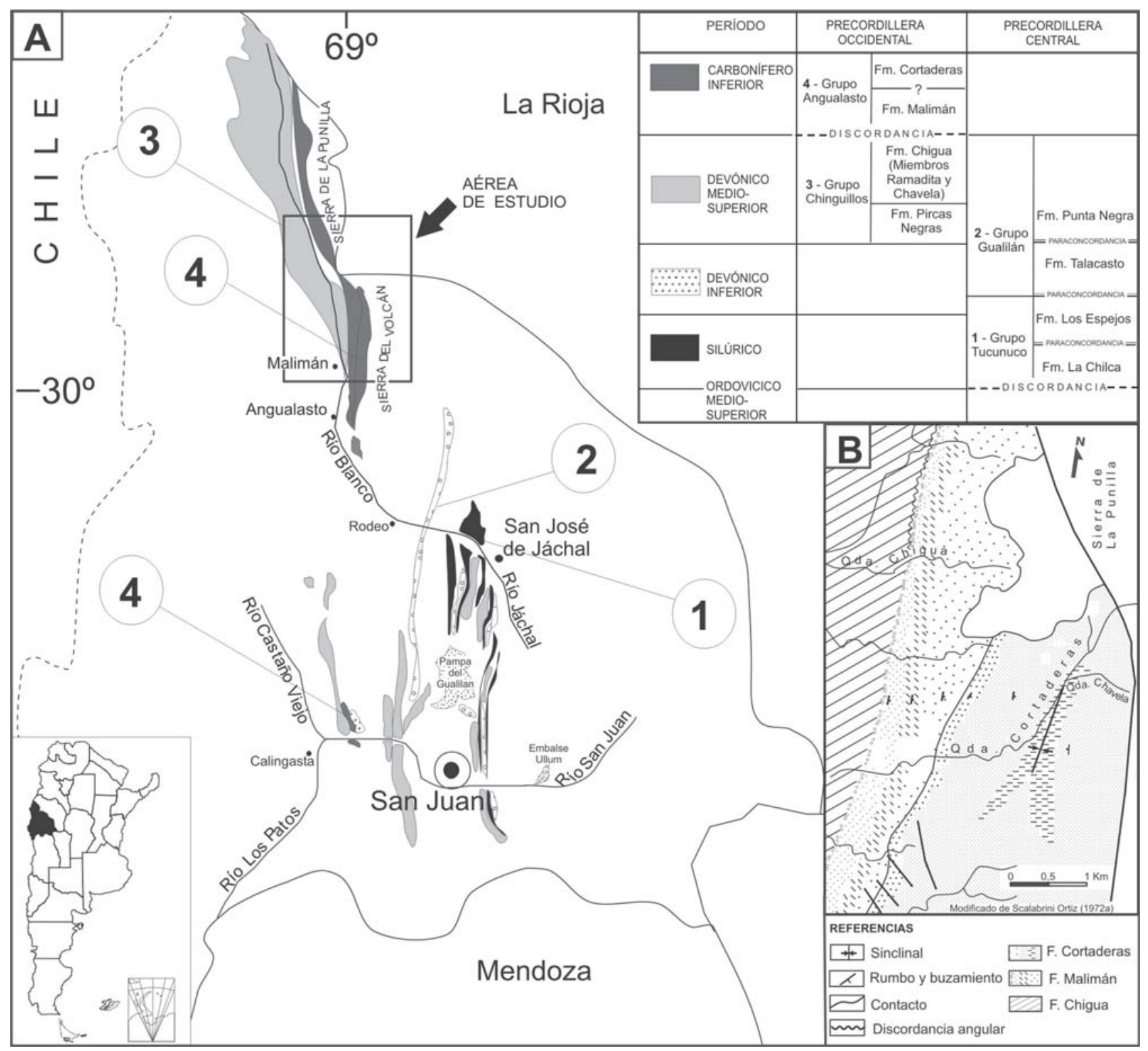

Figura 1. Area estudiada. A, ubicación y mapa geológico del área en la provincia de San Juan, y ubicación estratigráfica de las unidades mencionadas; $\mathbf{B}$, detalle de mapa geológico con la ubicación de la quebrada Cortaderas.

Figure 1. Study area. A, Location and geological map of the in San Juan province and stratigraphic location of the units mentioned; B, Detail of the geological map with the Cortaderas creek location. 
Paulophyton, y la Biozona de invertebrados Protocanites scalabrinii-Paurorhyncha chavelensis, las cuales permiten asignar a la FM al Carbonífero Inferior (Azcuy et al., 2000).

\section{ASOCIACIONES PALINOLÓGICAS}

La asociación autóctona de la FM contiene miosporas con una preservación buena a regular, y está acompañada por palinomorfos que han sido interpretados como retrabajados de sedimentos más antiguos. Las especies de palinomorfos autóctonos y retrabajados presentes en la FM, reunidos en grandes grupos sistemáticos, está listada en el Apéndice.

\section{CARACTERÍSTICAS DE LOS PALINOMORFOS RETRABAJADOS}

El conjunto de formas retrabajadas comprende esporas y paleomicroplancton (acritarcas, prasinoficeas y escasos quitinozoarios) los cuales presentan una preservación muy variada, desde formas muy bien preservadas hasta otras que se encuentran oxidadas y fragmentadas.

Se reconocen elementos retrabajados en todos los niveles analizados de la FM, con un rango variable entre $12,5 \%$ y $55 \%$ del total de la asociación (Figura 2). En su mayoría fueron clasificados a nivel específico lo cual permitió discriminarlos

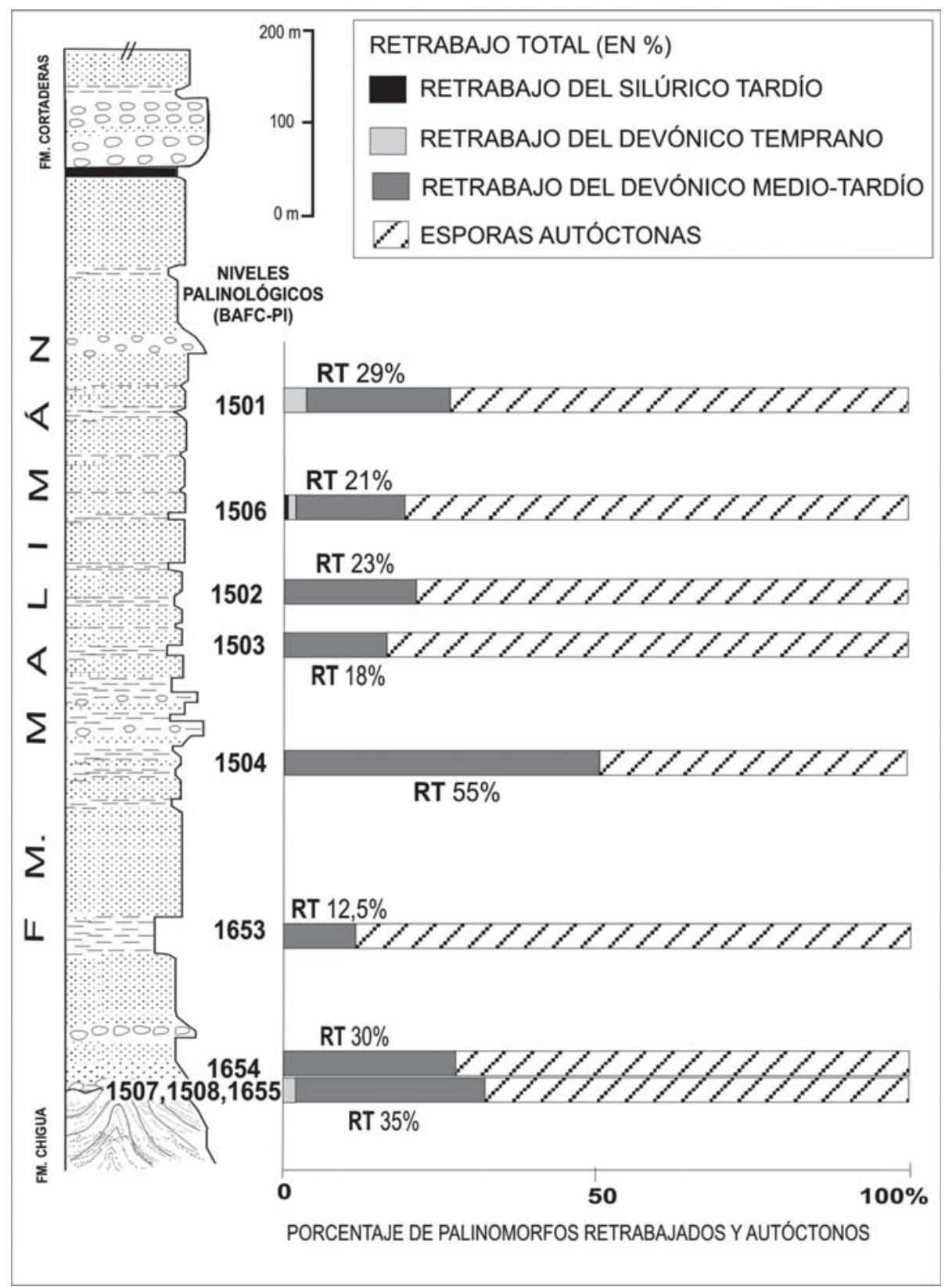

Figura 2. Porcentaje de palinomorfos retrabajados y autóctonos por cada nivel palinológico analizado (BAFC-PI) indicados en una columna estratigráfica. RT = porcentaje de retrabajo total.

Figure 2. Percentage of reworked and autochthonous palynomorphs per palynological level (BAFC-PI) showed in a stratigraphic column. $\mathrm{RT}=$ percentage of the total reworking. 


\begin{tabular}{|c|c|c|c|c|c|c|c|c|c|c|c|c|c|}
\hline SISTEMA & \multicolumn{4}{|c|}{ SILÚRICO } & \multicolumn{7}{|c|}{ DEVONICO } & & 忽 \\
\hline SERIE & \multicolumn{2}{|c|}{ TEMPR. } & \multicolumn{2}{|c|}{ TARDÍO } & \multicolumn{3}{|c|}{ TEMPRANO } & \multicolumn{2}{|c|}{ MEDIO } & \multicolumn{3}{|c|}{ TARDÍO } & 㝰 \\
\hline PISO & 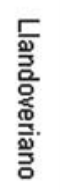 & 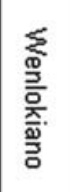 & 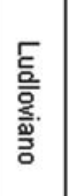 & $\begin{array}{l}\text { 물 } \\
\underline{\underline{\underline{0}}}\end{array}$ & 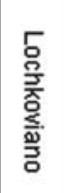 & 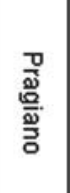 & $\begin{array}{l}\frac{m}{3} \\
\frac{3}{3} \\
\frac{2}{3} \\
\frac{3}{0}\end{array}$ & 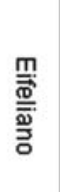 & 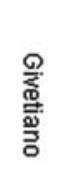 & 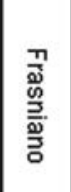 & & 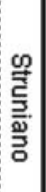 & 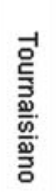 \\
\hline $\begin{array}{l}\text { Baltisphaeridium aniae }{ }^{*} \\
\text { Archeozonotriletes chulus } \\
\text { Onondagaella asymmetrica } \\
\text { Emphanisporites micrornatus } \\
\text { Cyclogranisporites plicatus } \\
\text { Polyedryxium embudum } \\
\text { Apiculiretusispora brandtii } \\
\text { Grandispora pseudoreticulata } \\
\text { Emphanisporites annulatus } \\
\text { Cordobesia uruguayensis }{ }^{*} \\
\text { Dictyotriletes subgranifer } \\
\text { Dibolisporites eifelensis }{ }^{*} \\
\text { Apiculatasporites microconus } \\
\text { Granulatisporites numinensis } \\
\text { Verrucosisporites scurrus } \\
\text { Acinosporites acanthomammillatus } \\
\text { Dictyotidium munificum } \\
\text { Geminospora lemurata } \\
\text { Stellinium comptum } \\
\text { Arkonites bilixus } \\
\text { Ancyrospora ampulla } \\
\text { Hemiruptia legaultii } \\
\text { Samarisporites triangulatus } \\
\text { Acinosporites ledundae } \\
\text { Polygonium barredae } \\
\text { Ammonidium garrasinoi } \\
\text { Cymatiosphaera perimembrana } \\
\text { Chomotriletes vedugensis } \\
\text { Pseudolunulidia imperatrizensis } \\
\text { Maranhites mosesii } \\
\text { Gorgonisphaeridium condensum } \\
\text { Acinosporites eumammillatus }\end{array}$ & & & & & & & W & & & , & & - & \\
\hline
\end{tabular}

Figura 3. Rango estratigráfico de especies seleccionadas de palinomorfos retrabajados reconocidas en la Formación Malimán (Carbonífero Temprano) indicando la edad de los conjuntos retrabajados. Las líneas punteadas indican registros dudosos. El asterisco (*) indica "cf". Las referencias correspondientes al Silúrico y Devónico se basan en los registros conocidos principalmente en América del Sur (Argentina, Brasil, Bolivia y Paraguay), y en el resto del mundo (especies indicadas en negrita).

Figure 3. Stratigraphic ranges of selected palynomorphs species recognized in the Malimán Formation (Early Carboniferous) indicating the age of reworked assemblages. Dashed lines indicate doubtful records. Asterisks (*) indicate "cf". References for Silurian and Devonian stratigraphic ranges are based mainly on well-known South American records (Argentina, Brazil, Bolivia and Paraguay), and occurrences from the rest of the world (species indicated in bold). 
del conjunto autóctono a partir de los rangos estratigráficos previos de estas especies halladas en cuencas siluro-devónicas de la región de Precordillera (Argentina) y de otras cuencas coetáneas en América del Sur y del resto del mundo. De esta manera, basado en la comparación con asociaciones de la región de Precordillera (Figura 1A), se reconocen dos conjuntos de palinomorfos atribuibles a dos intervalos: Devónico Temprano (Emsiano) y Devónico Medio-Tardío (Givetiano-Fameniano temprano). Un tercer conjunto es atribuido con dudas al Silúrico Tardío (Figuras 2-5). El conjunto mejor representado es el que corresponde al Devónico MedioTardío, mientras que los restantes, del Devónico Temprano y Silúrico Tardío, comprenden pocas especies (Figuras 2-4).

\section{Posible asociación del Silúrico (Ludloviano)}

En un único nivel de la sección estudiada (slide BAFC-Pl 1506 de la colección de palinología de la Universidad de Buenos Aires) y con una baja proporción (menos del 1\%) y diversidad de especies, se encuentra un conjunto de formas atribuidas al Silúrico (Figura 2). Si bien está muy poco representado, la presencia de las especies diagnósticas $O$. asymmetrica y $B$. sp. cf. B. aniae en el conjunto retrabajado (Figuras 3 y 4) sugieren un retrabajo de sedimentitas de esta edad. Ambos taxones han sido reconocidos en sedimentos silúricos del área de la Precordillera Central, Argentina (Figura 1A), ya que en la sección inferior de la Formación Los Espejos, de edad Ludloviano inferior (Pöthe de Baldis, 1981), se registró B. sp. cf. $B$. aniae; mientras que en la sección superior de esta misma unidad, la especie $O$. asymmetrica fue mencionada para la Asociación 5, de edad ludloviana (Rubinstein \& Brussa, 1999).

En otras cuencas de América del Sur, B. aniae fue reconocida como elemento característico en la Formación Tianguá (Cuenca Parnaíba, Brasil), del Llandoveriano medio (Rubinstein, 1997). Por su parte, O. asymmetrica fue citada en la Formación Kirusillas, en la región de Cochabamba, Bolivia, en la Zona I correspondiente al Ludloviano (Kimyai, 1983).

\section{Asociación del Devónico Temprano (Emsiano)}

Sólo tres niveles (BAFC-Pl 1508, 1506, 1501) de la FM contienen elementos retrabajados del Emsiano, entre el 1,3 y $3,7 \%$ del total de palinomorfos según el nivel considerado (Figura 2). Las siguientes especies de esporas constituyen los elementos diagnósticos que permiten asignar la asociación a esa edad: E. micrornatus, E. annulatus, D. sp. cf. D. eifeliensis y $D$. subgranifer. Otras especies que acompañan a este conjunto son: C. plicatus, A. lindlarensis, A. microconus y $A$. chulus (Figuras 3 y 4). Cabe señalar que las especies de microplancton halladas en la FM, tales como $M$. ramusculosum, E. arca, P. pharaonis, V. trispinosum, V. lairdii, V. polyaster, $D$. remota, $Q$. granulatus, $Q$. variabilis, tienen amplios rangos estratigráficos a nivel mundial abarcando gran parte del Devónico (Figura 4). Por lo tanto pueden ser especies que acompañen a cualquiera de los conjuntos retrabajados del Devónico aquí tratados.

Las especies diagnósticas del Emsiano citadas más arriba, son reconocidas en estratos del Devónico Temprano en cuencas del todo el mundo (e.g., Richardson \& McGregor, 1986; Streel et al., 1987; Melo \& Loboziak, 2003), y en particular
E. annulatus es un importante marcador estratigráfico a nivel global con surgimiento en el Emsiano temprano (Richardson \& McGregor, 1986; Streel et al., 1987). Este conjunto retrabajado se correlacionaría con la parte inferior de la Zona de Asociación E. annulatus-C. sextantii de Richardson \& McGregor (1986).

El Devónico Temprano de Argentina, muy escaso palinológicamente, se conoce en el área de la Precordillera Central, en la sección inferior de la Formación Talacasto (Figura 1A). La asociación palinológica proveniente de estos sedimentos contiene las esporas D. emsiensis (Allen) McGregor 1973 y D. echinaceus (Eisenack) Richardson emend. McGregor 1973, las cuales permitieron atribuir al conjunto al Lochkoviano superior-Emsiano (Le Hérissé et al., 1997). Dado que estas especies diagnósticas no están presentes en el retrabajo de la FM, su comparación resulta difícil. Los únicos elementos en común entre las dos asociaciones son: A. lindlarensis, A. chulus, E. rotatus y $S$. octoaster, especies de rango más longevo.

En Brasil, el surgimiento de E. annulatus es aún incierto debido a que los estratos del Emsiano temprano son desconocidos o están pobremente caracterizados palinológicamente. Esto ocurre para la Cuenca Amazonas y Parnaíba (Loboziak \& Melo, 2002), mientras que en la Cuenca Paraná, en la Formación Ponta Grossa (Miembro Jaguariaíva, parte superior), Dino (1999) presenta una asociación de esporas, Asociación 3, y entre ellas designa como Emphanisporites cf. erraticus a formas de E. annulatus para caracterizar el intervalo atribuido al Emsiano-Eifeliano (Dino, 1999; Loboziak \& Melo, 2002). En esta asociación del Emsiano temprano, se registran como primera aparición $E$. annulatus y otras formas como $E$. rotatus, $A$. (=Tholisporites) chulus, $D$. sp. cf. D. eifeliensis, $C$. plicatus, A. brandtii, $Q$. variabilis y $Q$. granulatus, y la ausencia de formas zonadas/cavadas como Samarisporites/ Grandispora, por lo que resulta comparable al conjunto aquí atribuido como retrabajado del Devónico Temprano (Figura 2).

En la Cuenca Amazonas, al Miembro Jatapu de la Formación Maecurú, se le asigna una edad Lochkoviano tardío-Emsiano temprano (Melo \& Loboziak, 2003), debido a que contiene elementos del Devónico Temprano más bajo, e.g. D. emsiensis (Allen) McGregor 1973, junto a V. polygonalis Lanninger 1968) y también, hacia el tope del Miembro, se registran ejemplares corroidos probablemente atribuidos a $D$. subgranifer. Esta es la especie diagnóstica de la Zona de Intervalo $D$. subgranifer (Su), restringida al Emsiano temprano (Melo \& Loboziak, 2003). El conjunto de elementos retrabajados hallados en la FM es comparable con la microflora registrada cerca del tope del Miembro Jatapu.

En Uruguay, la Formación Cordobés, del Lochkoviano tardío-Emsiano (Pöthe de Baldis, 1978; Grahn, 2003), tiene en común con el conjunto retrabajado a $C$. uruguayensis, cuyo rango estratigráfico se extiende hasta el Devónico Medio (Rubinstein, 1999; Quadros, 1999).

En Bolivia, la parte superior de la Formación Santa Rosa, del Lochkoviano tardío-Pragiano temprano (Racheboeuf et al., 1993; Blieck et al., 1996; Melo, 2000; Grahn, 2002), se caracteriza por la aparición de Dibolisporites spp. y A. microconus (McGregor, 1984), ambas reconocidas en el retrabajo de la FM. 


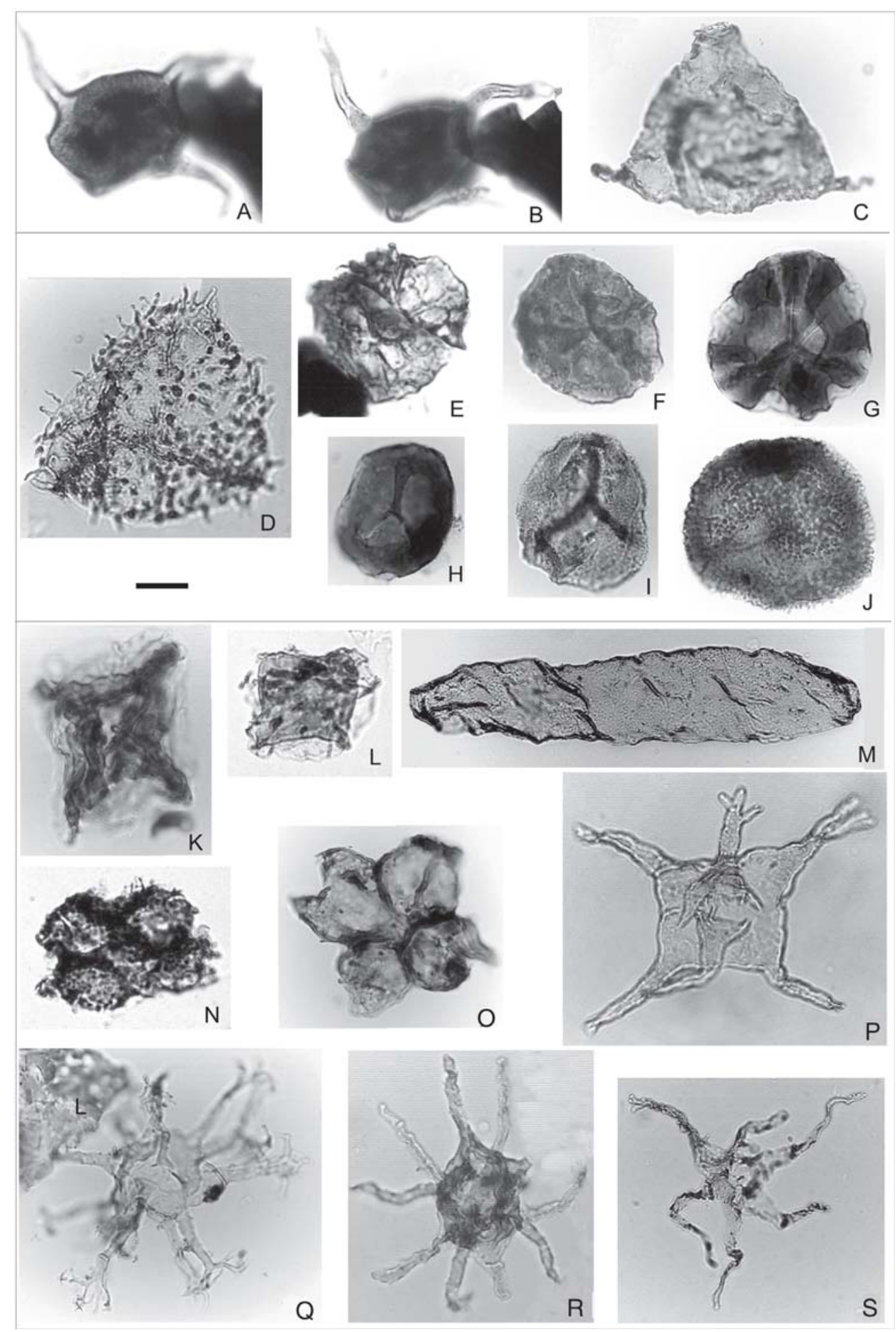

Figura 4. Asociaciones del Silúrico Tardío y Devónico Temprano y especies del Devónico s.l. (en paréntesis se indican el número de los preparados y la coordenada England Finder). A-C, Asociación del Silúrico Tardío: Baltisphaeridium sp. cf. B. aniae (A-B, BAFC-PI 15061, F20/4) y Onondagaella asymmetica (C, 1506-5, X50); D-J, Asociación del Devónico Temprano (Emsiano): D, Dibolisporites cf. D. eifeliensis (1508-5, J47/1); E, Dictyotriletes subgranifer (1506-2, 23); F,Emphanisporites micornatus (1506-1, X50); G,E. annulatus (1508-4, 26/3), H, Archaeozonotriletes chulus (1508-4, K42); I, Cyclogranisporites plicatus (1508-4, J47/2) y J,Acinosporites lindlarensis (1508-4, T29/3); K-R, Especies del Devónico (s.l.): K, Polyedryxium embudum (1655-2, Z34/1); L, Duvernaysphaera angelae (1655-1, T26/4); M,Navifusa bacilla (1655-2, O53); N, Quadrsiporites variabilis (1508-5, Q27); O,Q. granulatus (1508-4, K25/4); P, Exochoderma arca (I508-5, S29), Q, Multiplicisphaeridium ramusculosum (1655-2, B30/2), R, Diexallophasis remota (1655-2, Z35); S, Palacanthus ledanoisii (1655-1, D33/ 3). Escala para M, P, S $=20 \mu \mathrm{m}$; para A-L, N, O, Q-S = $15 \mu \mathrm{m}$.

Figure 4. Late Silurian and Early Devonian assemblages and Devonian (s.I.) species (in parenthesis are the slide number and the England Finder coordinates). A-C, Late Silurian assemblage; D-J, Early Devonian (Emsian);K-R, Devonian (s.I.) species. Scale bar: M, P, S = 20 um; 1 in $5 \mu \mathrm{m}$ : A-L, N, O, Q-S = $15 \mu \mathrm{m}$. 


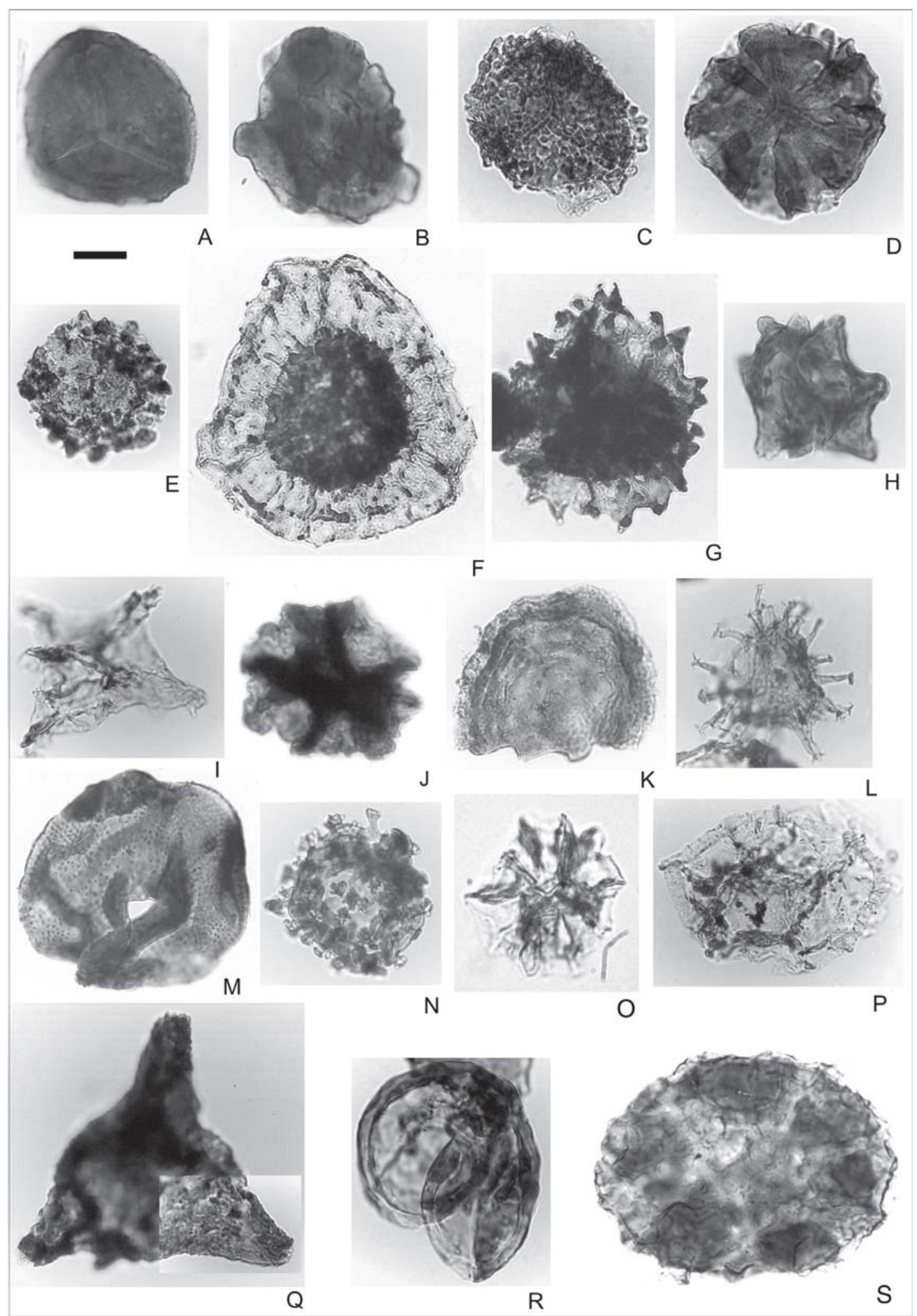

Figura 5. Asociación del Devónico Medio-Tardío (en paréntesis se indican el número de los preparados y las coordenadas England Finder). A, Geminospora lemurata (BAFC-PI 1501-1, K58/4); B, Verrucosisporites premnus (1503-1, Y29); C, Acinosporites eumamillatus (1508-4, Z50); D, Emphanisporites rotatus (1508-2, R56/4); E, Verrucosisporites scurrus (1508-1, O27/2); F, Grandispora pseudoreticulata (1508-4, P44); G, Ancyrospora ampulla (1506-1, X45); H, Cordobesia sp. cf. C. uruguayensis (1506-3, Z25/2); I, Stellinium comptum (1508-4, N29/2); J, Arkonites bilixus (1655-2, K35/3); K, Chomotriletes vedugensis (1503-1, C55/3; L, Ammonidium garrasinoi (1655-2, B56/1); M, Pseudolunulidia imperatrizensis (1506-1, G20/2); N, Umbellasphaeridium deflandrei (1506-4, G31); O, Cymatiosphaera perimembrana (1508-5, A37/4); P, Cymatiosphaera canadensis (1655-1, X54/2); Q. Estiastra rhytidoa (1508-2, K30); R, Hemiruptia legaultii (1508-5, X29/3); S, Maranhites mosesii (1506-2, C50/4). Escala: F-G, Q-R = $20 \mu \mathrm{m}$; A-E, G-P, S $=15 \mu \mathrm{m}$.

Figure 5. Middle-Late Devonian assemblage (in parenthesis are the slide number and the Enland Finder coordinate). Scale bar: F-G, Q-R $=20 \mu \mathrm{m} ; \mathrm{A}-\mathrm{E}, \mathrm{G}-\mathrm{P}, \mathrm{S}=15 \mu \mathrm{m}$. 
Por su parte, la Formación Icla, del Pragiano-Eifeliano temprano (McGregor, 1984; Racheboeuf et al., 1993; Melo, 2000; Grahn, 2002), incluye a E. annulatus, Dibolisporites spp., $A$. microconus, $A$. lindlarensis y una especie similar a $A$. acanthomammillatus las cuales también fueron reconocidas en el conjunto retrabajado.

\section{Asociación del Devónico Medio-Tardío (Givetiano-Fameniano temprano)}

Todos los niveles analizados de la FM contienen una diversificada y abundante cantidad de especies de esporas y microplancton retrabajadas del Devónico Medio-Tardío (Givetiano-Fameniano temprano), variable entre el 2,5 y el 55\% de la asociación palinológica (Figura 2). Las especies diagnósticas halladas incluyen un conjunto que correspondería al Givetiano-Frasniano como las especies cosmopolitas $V$. scurrus, característica desde el Eifeliano hasta el Frasniano temprano, G. lemurata, cuyo surgimiento marca el inicio del Givetiano, S. triangulatus, con surgimiento levemente más joven que la anterior y G. pseudoreticulata la cual es una especie endémica del Devónico Medio de América del Sur. Acompañan a esta asociación formas de microplancton tales como E. rhytidoa, $H$. legaultii y $C$. sp. cf. C. uruguayensis (Figura 5). Especies de rangos más jóvenes, Frasniano y Fameniano, incluyen: A ampulla, A. eumammillatus, $A$. ledundae, y entre el microplancton las especies $C$. perimembrana, C. canadensis, A. garrasinoi, U. deflandrei, G. condensum, M. mosesii, C. vedugensis y P. imperatrizensis (Figura 5).

En Argentina, en el área de la Precordillera Central, en la Formación Punta Negra (Figura 1A), se reconocen dos asociaciones A1 y A2, del Devónico Medio (EifelianoGivetiano) y del límite Givetiano/Frasniano respectivamente (Rubinstein, 1999), las cuales son comparables al conjunto retrabajado de la FM. Las especies compartidas con la A1 son: C. uruguayensis, $N$. bacilla, V. polyaster, D. munificum ( $=M$. munificus), C. canadensis, Quadrisporites sp., E. annulatus y $V$. scurrus y con la A2, E. rhytidoa, A. bilixus y M. mosesii.

La Formación Los Monos, al norte de Argentina, localmente de edad Givetiano tardío-Frasniano temprano (Ottone, 1996; Grahn, 2002), presenta numerosas especies en común, entre las que se pueden citar: A. nitida, V. scurrus, A. ledundae, $E$. rotatus, $S$. triangulatus, G. lemurata, G. pseudoreticulata, G. protea, A. bilixus, D. angelae, D. munificum, N. bacilla, E. rhytidoa, M. ramusculosum, E. arca, P. ledanoisii, P. embudum, P. pharaonis, S. comptum, S. octoaster, S. micropolygonale, Q. granulatus, V. trispinosum, V. polyaster, V. lairdii, A. garrasinoi, D. remota, P. barredae, G. condensum, $P$. imperatrizensis, U. deflandrei, C. vedugensis, H. legaultii, $M$. mosesii y M. brasiliensis (Figuras 4 y 5).

En Bolivia, la parte superior de la Formación Tequeje, de edad Givetiano tardío-Frasniano temprano (Ottone \& Rosello, 1996), contiene los siguientes elementos en común con el conjunto retrabajado aquí estudiado: A. ledundae, V. scurrus, G. lemurata, G. pseudoreticulata, $Q$. variabilis, D. angelae, $H$. legaultii, V. polyaster, V. trispinosum, U. deflandrei, A. garrasinoi, M. ramusculosum y N. bacilla. También en Bolivia, las muestras M-7 y M-8 analizadas por Pérez Leyton (1991), contienen algunas formas halladas en el retrabajo de la FM. En la M-7 aparece por primera vez G. lemurata, que marca el límite Eifeliano/Givetiano. La M-8 se caracteriza por la aparición de $S$. triangulatus, y la proliferación de las especies verrucosas y patinadas (V. premnus, V. scurrus, A. variabilis) asociadas con esporas grandes zonadas-pseudosacadas, lo cual permite asignarla al Givetiano tardío. En Paraguay, el pozo Picuiba brindó una asociación de esporas con la cual comparte principalmente a G. pseudoreticulata (Menéndez \& Pöthe de Baldis, 1967) y otras especies como C. perimembrana, $M$. brasiliensis, M. ramusculosum y N. bacilla, de edad Devónico Medio-Tardío (Pöthe de Baldis, 1979).

En la Cuenca Amazonas, Brasil, la Zona de Intervalo $G$. lemurata-Ch. ex gr. ligurata ( $L L i)$ del Givetiano temprano, que corresponde a la casi totalidad de la Formación Ereré (Melo \& Loboziak, 2003), tiene en común especies como $A$. macrospinosus, G. mammillata, V. scurrus, A. variabilis; y también $V$. premnus, especie que aparece en la Zona anterior (Zona Per) del Eifeliano temprano tardío. La siguiente Zona de Intervalo S. triangulatus (Trg), del Givetiano temprano tardíoFrasniano temprano, se define por la aparición de este taxón, asociado con especies de esporas patinadas como C. catillus y C. cyathus presentes en regiones del oeste de Gondwana (Loboziak \& Melo, 2002), las cuales también se registran en el retrabajo de la FM. El resto de las biozonas reconocidas en la Cuenca Amazonas por Melo \& Loboziak (2003) no son comparables pues se basan en especies de esporas mientras que el retrabajo de la FM del Devónico Tardío está representado especialmente por microplancton. La composición del conjunto retrabajado es semejante también a la asociación 5 de la Formación Ponta Grossa (Cuenca Paraná, Brasil), del Eifeliano tardío-Givetiano tardío (Dino, 1999), ya que comparten las especies A. macrospinosus, A. acanthomammillatus, $G$. mammillata, V. premnus, V. scurrus, G. lemurata y $S$. triangulatus. La asociación 6 de la misma unidad, atribuida al Givetiano tardío-Frasniano temprano, comparte las especies A. eumammillatus, Maranhites spp. y Pseudolunulidia spp., las cuales son abundantes en esta asociación.

\section{PROVENIENCIA DE LOS PALINOMORFOS RETRABAJADOS}

La paleogeografía de las áreas sedimentarias emergentes Siluro-Devónicas de cuencas argentinas en la Precordillera, en la provincia de San Juan (Figura 1A), indica tres posibles áreas fuentes para el material retrabajado: Formación Los Espejos, Formación Chigua y Formación Punta Negra.

\section{Formación Los Espejos}

El Silúrico de la Precordillera Central se extiende desde el norte del río Jáchal hasta el sur del río San Juan y se reconoce con la denominación de Grupo Tucunuco (Baldis \& Peralta, 2000) (Figura 1A), el cual suprayace en discordancia erosiva a distintas unidades del Ordovícico Medio y/o Superior, relacionado con la glaciación del Asghiliano tardío y los consecuentes cambios del nivel del mar (Rubinstein, 2001). 
Este conjunto de sedimentitas marinas depositadas en un ambiente de platafoma dominada por tormentas, incluye las Formaciones La Chilca, del Odovícico tardío-Silúrico temprano y Los Espejos, atribuida al Ludloviano temprano y Devónico Temprano (Figura 1A) en su parte superior (Rubinstein, 2001). Esta última sobreyace paraconcordantemente a la Formación La Chilca, y su techo está cubierto, también en paraconcordancia, por depósitos pelíticos de la Formación Talacasto, del Devónico Inferior.

La información palinológica de los depósitos silúricos de estas unidades es muy vasta, la cual ha sido obtenida de diversas localidades (e.g., Pöthe de Baldis, 1981; Rubinstein, 1989a, 1989b, 1997, 2001). En particular, el Ludloviano es el período mejor representado y más estudiado, debido a que contiene las asociaciones palinológicas más diversas y mejor preservadas, las cuales han sido correlacionadas con biozonas de graptolites (Rubinstein \& Brussa, 1999).

Como se mencionó más arriba, resulta prematuro confirmar la existencia de un retrabajo del Silúrico en la FM, ya que son muy pocas las formas presentes. Sin embargo, la presencia de O. asymmetrica (Figura 4C), un elemento diagnóstico encontrado en la Formación los Espejos, permite inferir que sedimentitas silúricas, aunque restringidas arealmente, estuvieron expuestas a la erosión durante la sedimentación carbonífera. Además, la Asociación 5 definida en la parte superior de la Formación Los Espejos (Rubinstein \& Brussa, 1999), es comparable a este posible conjunto retrabajado de edad silúrica no sólo por la presencia de esta especie diagnóstica que tiene su primera aparición en esta asociación, sino también por su baja diversidad. Asimismo, en la FM se registra una forma muy similar a $B$. sp. cf. $B$. aniae (Figuras 4A,B), hallada en la sección inferior de la Formación los Espejos (Ludloviano inferior, Pöthe de Baldis, 1981), la cual presenta algunas diferencias morfológicas con la especie definida originalmente para el Silúrico de África. Por lo tanto, es probable que la Formación Los Espejos haya sido otra área fuente de la FM.

\section{Formación Chigua}

En la Precordillera Occidental, aflora un conjunto de sedimentitas devónicas conocidas como Formaciones Chigua y Pircas Negras, que integran el Grupo Chinguillos (Baldis \& Peralta, 2000) (Figura 1). La Formación Chigua sobreyace en contacto tectónico a la Formación Pircas Negras, y su techo se encuentra en discordancia angular con la FM. En esta unidad se reconocen dos Miembros: Chavela (inferior) y Ramadita (superior), que se interpretan como una sucesión sedimentaria marina continua, con trilobites y restos de plantas que indican una edad givetiana-frasniana. La asociación palinológica obtenida del Miembro Chavela es atribuida al Devónico Medio (Rodríguez Amenábar et al., 2003; Amenábar et al., 2005), y contiene palinomorfos con una preservación buena a regular, cuyas especies más representativas son G. lemurata, Gr. pseudoreticulata, E. rotatus, V. scurrus, E. rhytidoa y $A$. bilixus. Estas mismas formas también se hallan presentes dentro del conjunto de elementos retrabajados en la FM debido a que las sedimentitas de la Formación Chigua actuaron como área fuente de palinomorfos durante el Carbonífero Temprano.
Otras especies presentes en la Formación Chigua de edad más antigua que el conjunto arriba mencionado, como $E$. annulatus, D. sp. cf. D. eifeliensis, D. subgranifer son probablemente formas retrabajadas del Devónico temprano (Emsiano) en el Devónico Medio (Amenábar et al., 2005) y también están presentes en el conjunto retrabajado de la FM.

\section{Formación Punta Negra}

En el sector de la Precordillera Central, el Devónico está representado por el Grupo Gualilán (Baldis \& Peralta, 2000) (Figura 1A), que incluye a las Fomaciones Talacasto y Punta Negra, del Lochkoviano temprano-Emsiano tardío y Devónico Medio-Superior? respectivamente, entre las las cuales existe una continuidad depositacional (Rubinstein, 2000). El Grupo Gualilán suprayace en discontinuidad estratigráfica al Grupo Tucunuco (Silúrico) y su techo está en contacto erosivo con depósitos carboníferos. La Formación Punta Negra es interpretada paleoambientalmente desde depósitos de turbiditas de un abanico submarino detrítico, a un fan delta, o un ambiente de aguas someras (véase Baldis \& Peralta, 2000). Los palinomorfos hallados en esta unidad fueron reunidos por Rubinstein (1999), en dos asociaciones, A1 y A2, atribuidas al Devónico Medio (Eifeliano-Givetiano) y Tardío (límite Givetiano-Frasniano) respectivamente. El conjunto retrabajado de la FM se asemeja a estas asociaciones no sólo en su contenido taxonómico sino también en cuanto al estado de preservación (regular), diversidad (baja) y cantidad de ejemplares (escasa). Además, su proximidad permite presumir que durante el Carbonífero Temprano esta unidad funcionó también como área fuente.

\section{CONCLUSIONES}

En base a la asociación palinológica analizada, se concluye que el conjunto retrabajado hallado en la FM abarca la mayor parte del Devónico, caracterizado por esporas del Devónico Temprano y Devónico Medio. El Devónico Tardío está representado en su mayoría por paleomicroplancton, probablemente debido a que en el Frasniano, la mayor parte del sur de América del Sur, estaba cubierta por mares escaseando las áreas vegetadas en esta región.

Asimismo, el Devónico cuspidal (Fameniano tardío o Struniano) no se encuentra registrado en la sección estudiada ya que Retispora lepidophyta (Kedo) Playford 1976, que caracteriza al Struniano, no ha sido hallada en el conjunto retrabajado analizado. Por lo tanto, se confirma un hiato erosivo y/o no depositacional en la FM, que comprende al Devónico Tardío (Fameniano tardío) y al Carbonífero Temprano (Tournaisiano). Esta marcada discontinuidad originada entre los sedimentos Devónicos y los depósitos Carboníferos en el flanco oeste de la Precordillera es producto de la colisión y acreción de un terreno alóctono (= bloque Chilenia) con la placa sudamericana ocurrida hacia fines del Devónico, lo que produjo la deformación de los depósitos siluro-devónicos (=Fase Chánica) y el levantamiento de la Protoprecordillera (Ramos et al., 1986). De esta manera, la presencia de palinomorfos retrabajados del Silúrico Tardío?-Devónico en las sedimentitas del Carbonífero Temprano, reafirma que luego del levantamiento 
de los terrenos Siluro-Devónicos ocurrió un importante período de erosión.

Por otro lado, se considera a la Protoprecordillera como el área fuente que aportó el material retrabajado durante el Carbonífero Temprano. A su vez, el estado de preservación de los elementos retrabajados es semejante al de las asociaciones de las cuales derivan, sugiriendo la proximidad del área fuente al depocentro (Richardson \& Rasul, 1978). Esto explicaría también la abundancia del material retrabajado principalmente del Devónico Medio-Tardío en la asociación de la FM.

Finalmente, se remarca que el estudio del retrabajo de palinomorfos permite además, tener un mayor conocimiento de las secuencias subyacentes de las cuales a veces no se conocen los depósitos in situ. La identificación de palinomorfos retrabajados en la asociación estudiada no fue una tarea fácil debido a la corta diferencia temporal existente entre los conjuntos autóctono y retrabajado. Sin embargo, su discriminación de las formas autóctonas es fundamental para realizar interpretaciones paleoambientales y bioestratigráficas más confiables (di Pasquo \& Azcuy, 1997) y el análisis de la proveniencia de los mismos constituye una herramienta más a la hora de abodar reconstrucciones paleogeográficas.

\section{AGRADECIMIENTOS}

La autora agradece a Mercedes di Pasquo y Carlos L. Azcuy, por todo el apoyo brindado durante la realización del trabajo, a José Henrique G. de Melo y Eduardo G. Ottone por los valiosos comentarios como árbitros de este trabajo, a la Universidad de Buenos Aires y al CONICET por el apoyo económico recibido a través del PIP 2307/00. Es una contribución al Proyecto 471.

\section{REFERENCIAS}

Amenábar, C.R.; di Pasquo, M.M.; Carrizo, H.A. \& Azcuy, C.L. 2005. Palynology of Chigua and Malimán Formations in the Sierra del Volcán, San Juan province, Argentina. Part I. Paleomicroplankton and acavate smooth and ornamented spores. Ameghiniana (en prensa).

Azcuy, C.L.; Carrizo, H.A. \& Caminos, R. 2000. Carbonífero y Pérmico de las Sierras Pampeanas, Famatina, Precordillera, Cordillera Frontal y Bloque San Rafael. In: R. Caminos (ed.) Geología Argentina, Instituto de Geología y Recursos Minerales, p. 261-318.

Baldis, B.A. \& Peralta, S.H. 2000. Silúrico y Devónico de la precordillera de Cuyo y Bloque de San Rafael. In: R. Caminos (ed.) Geología Argentina, Instituto de Geología y Recursos Minerales, p. 215-238.

Blieck, A.; Gagnier, P.-Y.; Bigey, E.P.; Edgecombe, G.D.; Janvier, P.; Loboziak, S.; Racheboeuf, P.R.; Sempere, T. \& Steemans, P. 1996. New Devonian fossil localities in Bolivia. Journal of South American Earth Sciences, 9:295-308.

Césari, S.N. \& Gutiérrez, P.R. 2000. Palynostratigraphy of Upper Paleozoic sequences in central-western Argentina. Palynology, 24:113-146. di Pasquo, M.M. \& Azcuy, C.L. 1997. Palinomorfos retrabajados en el Carbonífero Tardío de la Cuenca Tarija (Argentina) y su aplicación a la datación de eventos diastróficos. Revista da Universidade de Guarulhos, Geociências (número especial), 2:28-42.

Dino, R., 1999. Palynostratigraphy of the Silurian and Devonian sequence of the Paraná Basin, Brazil. In: ORDOVICIANDEVONIAN PALYNOSTRATIGRAPHY IN WESTERN GONDWANA: UPDATE, PROBLEMS AND PERSPECTIVES, 1999. Resúmenes expandidos, Rio de Janeiro, UERJ, p. 27-61.

Grahn, Y. 2002. Upper Silurian and Devonian Chitinozoa from central and southern Bolivia, central Andes. Journal of South American Earth Sciences, 15:315-326.

Grahn, Y. 2003. Silurian and Devonian chitinozoan assemblages from the Chaco-Paraná Basin, northeastern Argentina and central Uruguay. Revista Española de Micropaleontología, 35(1):18.

Kimyai, A. 1983. Palaeozoic microphytoplankton from South America. Revista Española de Micropaleontología, 15(3):415426.

Le Hérissé, A.; Rubinstein, C. \& Steemans, P. 1997. Lower Devonian Palynomorphs from the Talacasto Formation, Cerro del Fuerte Section, San Juan Precordillera, Argentina. Acta Universitatis Carolinae Geologica, 40:497-515.

Limarino, C.O. \& Césari, S.N. 1993. Reubicación estratigráfica de la Formación Cortaderas y definición del Grupo Angualasto (Carbonífero Inferior, Precordillera de San Juan). Revista de la Asociación Geológica Argentina, 47(1):61-72.

Loboziak, S. \& Melo, J.H.G. 2002. Devonian miospore successions of Western Gondwana: update and correlation with Southern Euroamerican miospore zones. Review of Palaeobotany and Palynology, 121:133-148.

McGregor, D.C. 1984. Late Silurian and Devonian spores from Bolivia Academia Nacional de Ciencias de Córdoba, Miscelánea, 69:1-43.

Melo, J.H.G. 2000. Palynological evaluation of some SilurianDevonian sections of southern Bolivia. In: CONGRESO GEOLÓGICO BOLIVIANO, 14, 2000. Memorias, La Paz, CGB, p. 136-141.

Melo, J.H.G. \& Loboziak, S. 2003. Devonian-Early Carboniferous miospore biostratigraphy of the Amazon Basin, Northern Brazil. Review of Palaeobotany and Palynology, 124:131-202.

Menéndez, C.A \& Pöthe de Baldis, E.D. 1967. Devonian Spores from Paraguay. Review of Palaeobotany and Palynology, 1:161172.

Ottone, E.G. 1996. Devonian palynomorphs from the Los Monos Formation, Tarija Basin, Argentina. Palynology, 20:101-151.

Ottone, E.G. \& Rossello, E.A. 1996. Palinomorfos devónicos de la Formación Tequeje, Angosto del Beni, Bolivia. Ameghiniana, 33(4):443-452.

Pérez Leyton, M. 1991. Miospores du Devonien Moyen et Superior de la coupe de Bermejo-La Angostura (Sud-Est de la Bolivie). Annales de la Société Géologique de Belgique, 113(2):373-389.

Pöthe de Baldis, E.D. 1978. Paleomicroplancton adicional del Devónico inferior de Uruguay. Revista Española de Micropaleontología, 9(2):235-250.

Pöthe de Baldis, E.D. 1979. Acritarcos y quitinozoos del Devónico Superior de Paraguay. Palinologia, 1:161-177.

Pöthe de Baldis, E.D. 1981. Paleomicroplancton y mioesporas del Ludloviano inferior de la Formación Los Espejos en el perfil Los Azulejitos, en la Provincia de San Juan, República Argentina. Revista Española de Micropaleontología, 2(13):231-265. 
Quadros, L.P.1999. Silurian-Devonian acritarch assemblages from Paraná Basin: an update and correlation with Northern Brazilian basins. In: ORDOVICIAN-DEVONIAN PALYNOSTRATIGRAPHY IN WESTERN GONDWANA: UPDATE, PROBLEMS AND PERSPECTIVES, 1999. Resúmenes expandidos, Río de Janeiro, UERJ, p. 105-145.

Racheboeuf, P.; Le Hérissé, A..; Paris, F.; Babin, C.; Guillocheau, F.; Truyols-Massoni, M. \& Suárez-Soruco, R. 1993. Le Dévonien de Bolivie: biostratigraphie et chronostratigraphie. Compte Rendus de l'Academie de Sciences de Paris, 317(2):795802.

Ramos, V.; Jordan, T.; Allmendinger, R.; Mpodozis, C.; Kay, S.; Cortes, J. \& Palma, M. 1986. Paleozoic terranes of the central Argentine-Chilean Andes. Tectonics, 5(6):855-880.

Richardson, J.B. \& McGregor, D.C. 1986. Silurian and Devonian spore zones of the Old Red Sandstone continent and adjacent regions. Geological Survey of Canada, 79 p. (Bulletin 364).

Richardson, J.B. \& Rasul, S.M. 1978. Palynological evidence for the age and provenance of the Lower Old Red Sandstone from the Apley Barn Borehole, Witney, Oxfordshire. Procedings of the Geological Association, 90(1):27-42.

Rodríguez Amenábar, C.; di Pasquo, M. \& Carrizo, H.A. 2003. El límite Devónico-Carbonífero en la Quebrada Cortaderas, provincia de San Juan, Argentina. In: SIMPOSIO ARGENTINO DE PALEOBOTÁNICA Y PALINOLOGÍA, 12, 2003. Resúmenes, Buenos Aires, p. 36-37.

Rubinstein, C.V. 1989a. Acritarcos del Silúrico del Valle del Río Jáchal (Formación Los Espejos), provincia de San Juan, República Argentina. Parte I: Subgrupos Acanthomorphitae y Sphaeromorphitae. Ameghiniana, 21(1-2):449-476.

Rubinstein, C.V. 1989b. Acritarcos del Silúrico del Valle del Río Jáchal (Formación Los Espejos), provincia de San Juan, República Argentina. Subgrupos Herkomorphitae, Netromorphitae y Polygonomorphitae. Ameghiniana, 26(1-2):83-100.

Rubinstein, C.V. 1997. Silurian acritarchs from South America: a review. Acta Universitatis Carolinae Geologica, 40:603-629.

Rubinstein, C.V. 1999. Primer registro palinológico de la Formación Punta Negra (Devónico Medio-Superior), de la Precordillera de San Juan, Argentina. In: SIMPOSIO ARGENTINO DE PALEOBOTÁNICA Y PALINOLOGÍA, 10, 1999. Atas, Buenos Aires, APA, p. 13-18.

Rubinstein, C.V. 2000. Middle Devonian Palynomorphs from the San Juan Precordillera, Argentina: Biostratigraphy and Paleobiogeography. In: CONGRESSO IBERICO DE PALEONTOLOGIA, 1, 2000. Libro de Resúmenes, Evora, Univesidade de Évora, p. 274-275.

Rubinstein, C.V. 2001. Microflora y microplancton silúricos de la Precordillera Central de San Juan, Argentina. In: M.A. Fombella Blanco; D. Fernández González \& R.M. Valencia Barrera (eds.) Palinologia: diversidad y aplicaciones, Secretariado de Publicaciones/Universidad de León, p. 45-54.

Rubinstein, C.V. \& Brussa, E.D. 1999. A palynomorph and graptolite biostratigraphy of the Central Precordillera Silurian Basin, Argentina. Bollettino della Società Paleontologica Italiana, 38(2/ 3):257-266.

Streel, M.; Higgs, K.; Loboziak, S.; Riegel, W. \& Steemans, P. 1987. Spore stratigraphy and correlation with faunas and floras in the type marine Devonian of the Ardenne-Rhenish regions. Review of Palaeobotany and Palynology, 50:211-229.

Received in January, 2006; accepted in March, 2006.
Apéndice. Especies de palinomorfos autóctonos y retrabajados presentes en la Formación Malimán.

Appendix. Autoctonous and reworked palynomorph species found in Malimán Formation.

\section{Asociación autóctona \\ Esporas}

Anapiculatisporites amplus Playford \& Powis 1979

Anapiculatisporites hystricosus Playford 1964

Apiculiretusispora semisenta (Playford) Massa et al., 1980

Convolutispora insulosa Playford 1978

Crassispora scrupulosa Playford 1971 emend. Playford \& Satterthwait 1988

Cristatisporites indignabundus (Loose) Potonié \& Kremp emend. Staplin \& Jansonius 1964

Densosporites anulatus (Loose) Schopf, Wilson \& Bentall 1944

Densosporites spinifer Hoffmeister, Staplin \& Malloy 1955

Densosporites spitsbergensis Hoffmeister, Staplin \& Malloy 1955

Dibolisporites medaensis (Playford) Playford 1976

Dibolisporites microspicatus Playford 1978

Grandispora debilis Playford 1971

Leiotriletes ornatus Ishchenko 1956

Pustulatisporites gibberosus (Hacquebard) emend. Playford 1964 Verrucosisporites baccatus Staplin 1960

Verrucosisporites congestus Playford 1964

Verrucosisporites morulatus (Knox) Smith \& Butterworth 1967

\section{Asociación de retrabajo \\ Esporas}

Acinosporites acanthomammillatus Richardson 1965

Acinosporites eumamillatus Loboziak, Streel \& Burjack 1988 (Figura 5C)

Acinosporites ledundae Ottone 1996

Acinosporites lindlarensis Riegel 1968 (Figura 4J)

Acinosporites macrospinosus Richardson 1965

Apiculatasporites microconus (Richardson) McGregor \& Camfield 1982

Ancyrospora ampulla Owens 1971 (Figura 5G)

Ancyrospora spp.

Apiculatisporis morbosus Balme \& Hassell 1962

Apiculiretusispora brandtii Streel 1964

Archaeozonotriletes chulus (Cramer) Richardson \& Lister 1969

(Figura 4H)

Archeozonotriletes variabilis Naumova emend. Allen 1965

Cyclogranisporites plicatus Allen 1965 (Figura 4I)

Cymbosporites catillus Allen 1965

Cymbosporites sp. cf. C. verrucosus Richardson \& Lister 1969

Dibolisporites sp. cf. D. eifeliensis (Lanninger) McGregor 1973 (Figura 4D)

Dictyotriletes subgranifer McGregor 1973 (Figura 4E)

Emphanisporites annulatus McGregor 1961 (Figura 4G)

Emphanisporites micrornatus Richardson \& Lister 1969 (Figura 4F)

Emphanisporites rotatus (McGregor) McGregor 1973 (Figura 5D)

Geminospora lemurata Balme 1962 (Figura 5A)

Grandispora mammillata Owens 1971

Grandispora protea (Naumova) Moreau-Benoit 1980

Grandispora pseudoreticulata (Menéndez \& Pöthe de Baldis)

Ottone 1996 (Figura 5F)

Granulatisporites numinensis Allen 1965

Hystricosporites spp.

Samarisporites triangulatus Allen 1965

Verrucosisporites premnus Richardson 1965

Verrucosisporites scurrus (Naumova) McGregor \& Camfield 1982

\section{Prasinophycea}

Cymatiosphaera canadensis Deunff 1961 (Figura 5P) Cymatiosphaera perimembrana Staplin 1961 (Figura 5O)

Dictyotidium munificum (Wicander \& Wood) Amenábar, di Pasquo, Carrizo \& Azcuy 2006(=Muraticavea munificus Wicander \& Wood 1981)

Duvernaysphaera angelae Deunff 1964 (Figura 4L)

Hemiruptia legaultii Ottone 1996 (Figura 5R)

Leiosphaeridia spp. 
Maranhites brasiliensis Brito 1965 emend. Burjack \& Oliveira 1989 Maranhites mosesii (Sommer) Brito emend. Burjack \& Oliveira 1989 Polyedryxium decorum Deunff 1955

Polyedryxium embudum Cramer 1964 (Figura 4K)

Polyedryxium pharaonis Deunff ex Deunff 1961

Pterospermella solis Wicander 1974

Tasmanites spp.

Acritarca

Ammonidium garrasinoi Ottone 1996 (Figura 5L)

Arkonites bilixus Legault 1973 (Figura 5J)

Baltisphaeridium sp. cf. B. aniae Jardin, et al. 1974 (Figura 4A- B)

Cordobesia sp. cf. C. uruguayensis (Machiavello) Pöthe de Baldis 1978 (Figura 5H)

Diexallophasis remota (Deunff) Playford 1977 (Figura 4R)

Estiastra barbata Downie 1963

Estiastra rhytidoa Downie 1963 (Figura 5Q)

Exochoderma arca Wicander \& Wood 1981 (Figura 4P)

Gorgonisphaeridium condensum Playford 1981

Multiplicisphaeridium ramusculosum (Deflandre) Lister 1970

(Figura 4Q)

Navifusa bacilla (Deunff) Playford 1977 (Figura 4M)

Onondagaella asymmetrica (Deunff) Cramer 1966 (Figura 4C)

Palacanthus ledanoisii (Deunff) Playford 1977 (Figura 4S)

Polygonium barredae Ottone 1996

Pseudolunulidia imperatrizensis Brito \& Santos 1965 (Figura 5M)

Stellinium comptum Wicander \& Loeblich 1977 (Figura 5I)

Stellinium octoaster (Staplin) Jardiné, Combaz, Magloire, Peniguel

\& Vachey 1972

Stellinium micropolygonale (Stockmans \& Willière) Playford 1977

Umbellasphaeridium deflandrei (Moreau-Benoit) Jardiné et al. 1972

(Figura 5N)

Verhyachium downiei Stockmans \& Willière 1962

Verhyachium lairdii Deflandre 1946 ex Deunff 1959

Verhyachium polyaster Staplin 1961

Verhyachium trispinosum (Eisenack) Deunff 1954

\section{Chlorophycea}

Chomotriletes vedugensis Naumova 1953 (Figura 5K)

Quadrisporites granulatus (Cramer) Ströther 1991 (Figura 4O)

Quadrisporites variabilis (Cramer) Ottone \& Rossello 1996 (Figura 4N) 